\title{
Recursos naturales prehispánicos: el guano de isla en Moquegua
}

\author{
Pre-Hispanic natural resources: the island guano in Moquegua
}

ORCID: 0000-0002-8689-7108

\section{RESUMEN}

El guano es un recurso natural que ha jugado un papel relevante en la historia del Perú. No obstante, la mayoría de estudios se han reducido con bastante razón al siglo XIX, cuando el país tuvo bonanza económica gracias a este requerido fertilizante que conquistó los mercados europeos y norteamericanos. Por ese motivo, este artículo se orienta a las épocas más ancestrales, con registros referentes a los periodos prehispánico y colonial, hallados en crónicas y otras fuentes documentales. Para esta ocasión, tomamos como ejemplo el caso de Moquegua, región que cuenta con un litoral rico en depósitos de guano, cuyas noticias más antiguas contienen informaciones referentes a la utilización de dicho recurso, a las posesiones de las comunidades altoandinas y a las posteriores disputas emprendidas por los colonos españoles.

Palabras clave: Guano, Moquegua, recursos prehispánicos.

\section{ABSTRACT}

Guano is a natural resource that has played a significant role in the history of Peru. However, most studies have been quite rightly reduced to the 19 th century, when the country had economic bonanza thanks to this required fertilizer that conquered the European and North American markets. For this reason, this article is geared towards the most ancient times, with records relating to the pre-Hispanic and colonial periods, found in chronicles and other documentary sources. For this occasion, we take as an example the case of Moquegua, region that has a coastline rich in guano deposits, whose oldest news contains information concerning the use of such a resource, to the possessions of the Upper Andean communities and to the subsequent disputes undertaken by the Spanish settlers.

Keywords: Guano, Moquegua, pre-Hispanic resources.

Un tema poco investigado es el de los fertilizantes y abonos empleados en tiempos prehispánicos, por estar sus noticias diseminadas en crónicas, relaciones y documentos inéditos de archivo. Estos recursos tuvieron gran importancia desde antes de la invasión europea para el aumento de la producción agrícola. El acceso a los abonos debió constituir una preocupación para los pueblos andinos, tan dedicadas a las labores relacionadas con el agro; preocupación que se traduce en la construcción de andenes en todo el territorio, canales hidráulicos, chacras hundidas, etc. En todas estas manifestaciones del ingenio indígena, se observa el enorme interés y la necesidad de aumentar el rendimiento de la tierra

María Rostworowski (2005, p. 80).

1'Facultad de Educación, Comunicación y Humanidades. Universidad Nacional Jorge Basadre Grohmann. E-mail: pperaltac@unjbg.edu. 


\section{Introducción: El guano en la historia del Perú}

Se denomina guano al cúmulo de deyecciones de aves marinas. Este sustrato, rico en nitrógeno, urea, fósforo y otros compuestos, si bien logró prestigio internacional como abono durante el siglo XIX, ya era conocido desde el periodo prehispánico, probablemente hacia el año 750 a. C. Por lo que sabemos, las islas de Lobos, Macabí y Guañape fueron utilizadas por los mochicas para extraer el rico fertilizante, llegando a sacrificar varias jóvenes en honor a la luna y los dioses marinos. El guano también llegó a ser preciado en la época incaica, tal como refiere el cronista Garcilaso de la Vega, llegándose a castigar hasta con la muerte a quienes ingresaran a las islas guaneras en tiempo de la cría de las aves marinas (Tantaleán Arbulú, 2011).

Durante la colonia, los españoles comenzaron a reconocer la importancia de este recurso y, poco a poco, empezaron a ocupar las islas y a comerciar con el guano. Por ejemplo, en el Archivo de Moquegua consultamos una escritura referente a Ilo del 25 de febrero de 1733, siguiendo el derrotero que nos da Edwin Adriazola Flores (2015, p. 33), donde el maese Manuel de Lartiga del navío Concepción, a nombre de Juan Córdova Paniagua, vendió al general Diego de Hidalgo...

[...] una barcada de guano en dicho navio de tres mil y dosientas fanegas más o menos, puestas en el puerto de la Yerbabuena en qualquiera de los meses de maio o junio, julio, agosto del año que viene de mil setesientos treinta y quatro, al presio de once reales cada fanega cuia importancia se ha de obligar a pagarme como principal el general don Bernardo Carbonera y Sotomaior en esta forma, los dos mil y quinientos pesos de contado luego que se acabe de medir la dicha barcada de [...] guano en el dicho puerto de la Yerbabuena y la restante cantidad cumplimiento a la que montan el todo de la dicha barcada del dicho día último de dicha medida en ocho meses libres todas de carga y descarga, medida y otros gastos de dicho puerto de la Yerbabuena [...] (ARM, escribano Thomás de Valencia 1733, f. 253 r.).

Gracias a las investigaciones de estudiosos como Alexander von Humboldt y Mariano de Rivero, el guano logró obtener prestigio por sus cualidades fertilizantes. Fue a partir del año 1840, cuando el Perú ingresó a su "era del guano", comenzando la explotación y exportación de este recurso al mercado europeo y norteamericano. Según Gregory Cushman (2018), entre 1840 y 1879 “el Perú exportó aproximadamente 12,7 millones de toneladas métricas de guano de isla, equivalente a un valor de venta de entre 100 y 150 millones de libras esterlinas" (p. 94). Lo que le significó al país los recursos suficientes para emprender un camino de progreso, que al final terminó como una oportunidad desperdiciada.

Tabla 1. Depósitos de guano durante el decenio de 1870

\begin{tabular}{ll}
\hline & Covaderas durante el decenio de $\mathbf{1 8 7 0}$ \\
\hline Islas de Chincha & Punta Pandro \\
\hline Islas de Guañape & Punta Supe \\
\hline Islas de Macabí & Punta Chinos \\
\hline Islas Lobos de Tierra & Punta Mejillones \\
\hline Islas Lobos de afuera & Islotes Guanillos \\
\hline Islas Viejas (Bahía Independencia) & Islotes Los Pájaros o Caletas de Patillos \\
\hline Islas Lobillos & Islotes Colohue \\
\hline Islas San Gallán & Islotes Jesús \\
\hline Islas Blancas & Islotes Albisuri \\
\hline Islas Arica & Islote Zárate \\
\hline Islas Pachacamac & Islote Ballesta \\
\hline Islas Chao & Islote Palominos \\
\hline Islas Cornejos & Islote Pescadores \\
\hline Islas La Viuda & Islote Chiquitana \\
\hline Islas Tortuga & Islote Bravo \\
\hline Islas Coroco & Islote Mayorca \\
\hline Islas Corcobado & Islote Pelado \\
\hline Islas Ocona & Islote Carquin \\
\hline
\end{tabular}




\begin{tabular}{ll} 
Bahía Cipana & Ensenada Chiquitana \\
\hline Bahía Chomache & Ensenada Chimbote \\
\hline Bahía Samanco & Ensenada al Norte de Iquique (Pisagua) \\
\hline Bahía Ferrol & Quebrada Pica \\
\hline Punta Huanillo & Quebrada Camarones \\
\hline Punta Blanca & Pabellón Pica \\
\hline Punta de Lobos & Caleta Pabellón \\
\hline Punta Patachi & Cabo Lobos \\
\hline Punta Pinadores & Morro Arica \\
\hline Punta Alier & Cerro de Carreteras \\
\hline Punta Loboso & Hormigas Afuera \\
\hline Punta San Nicolás & La Cruz Medio \\
\hline Punta Dona María & Puerto de Lomas \\
\hline Punta Azua & La Pampa de Chochapampa \\
\hline Punta Chocalla & La Capilla \\
\hline Punta Solar & Chanabaya \\
\hline
\end{tabular}

Fuente: Tantaleán Arbulú, 2011.

Tras el desastre de la guerra del guano y el salitre (1879-1883) y la aparición de los fertilizantes sintéticos que desplazaron gradualmente al guano, culminó la primera era del guano. Fue a partir de 1909, con la creación de la Compañía Administradora del Guano (CAG), que comenzó la segunda era. Según registros de la CAG, durante la primera campaña (1915-1916), de Punta de Coles se extrajeron 85 toneladas de guano. En total, desde 1909 hasta 1963, año en que fue desarticulada la CAG, la compañía produjo ganancias por 435 millones de dólares, según valor del 2007 (Cushman, 2018).

Tras el final de la CAG, luego de pasar por siete instituciones nacionales, la administración del guano e islas guaneras finalmente recayó en Agrorural, dependencia del Ministerio de Agricultura y Riego. Paralelamente, con la creación de la Reserva Nacional del Sistema de Islas, Islotes y Puntas Guaneras (RNSIIPG) en 2009, 22 islas, islotes, grupos de islas y 11 puntas (entre ellas Punta de Coles) pasaron a la protección sostenible del Estado.

\section{El guano en la Moquegua prehispánica}

Como lo demuestran los distintos registros históricos y arqueológicos, los pueblos del antiguo Perú practicaron la agricultura desde épocas muy remotas. Esta actividad, que fue perfeccionándose con el tiempo, requería sin duda de fertilizantes. Con respecto a Moquegua, desde aproximadamente el año 1000 a. C. existen evidencias de que los habitantes de esta región incluyeron en sus plantaciones al maíz (Asociación Contisuyo, 1997), un producto que, en palabras del cronista Pedro Cieza de León (2005 [1553]), requería del guano de isla, [...] porque si dejan de echar deste estiércol cogen poco maíz [...] (p. 205).

Aunque es difícil averiguar cómo se utilizó el guano en el periodo preincaico, las informaciones referentes al Tahuantinsuyo arrojan algunas luces sobre el uso temprano de este recurso. En un pasaje de sus Comentarios Reales, Garcilaso de la Vega (2009 [1609]) menciona que:

[...] en la costa de la mar, desde más abajo de Arequipa hasta Tarapacá, que son más de doscientas leguas de costa, no echan otro estiércol sino el de los pájaros marinos, que los hay en toda la costa del Perú grandes y chicos, y andan en bandas tan grandes que son increíbles si no se ven. Crían en unos islotes despoblados que hay por aquella costa, y es tanto el estiércol que en ellos dejan, que también es increíble, de lejos parecen los montones del estiércol puntas de alguna sierra nevada [...] (p.240).

Más adelante, Garcilaso señala cómo era la administración de los cusqueños:

[...] En tiempos de los Reyes Incas habia tanta vigilancia en guardar aquellas aves, que al tiempo de la cría a nadie era lícito entrar en las islas, so pena de la vida, porque no las asombrasen y echasen de sus nidos. Tampoco era lícito el matarlas en ningún tiempo, dentro ni fuera de las islas, so la misma pena [...]poníanles mojones por que los de la una provincia no se entrasen en el distrito de la 
otra; y repartiéndola más en particular, daban en el mismo límite a cada pueblo su parte y a cada vecino la suya, tanteando la cantidad de estiércol que habia menester, y, so pena de muerte, no podía el vecino de un pueblo tomar estiércol del término ajeno, porque era hurto, ni de su mismo término podía sacar más de cantidad que le estaba tasada conforme a sus tierras, que le era bastante, y la demasía la castigaban por el desacato $[\ldots]$ (p. 240).

En efecto, cada natural tenía que tomar la cantidad de abono que le correspondía y hacer que este llegue hasta sus cultivos. Para transportar el guano desde las islas -tomando lo que conocemos de los primeros años de la colonia-se hacía uso de balsas, algunas de ellas hechas con cuero de lobo marino. Para el traslado desde las costas hasta los valles interiores se utilizaban los carneros de la tierra, como los españoles llamaban a las llamas (Pizarro \& Soto, 2017). Los registros más tempranos demuestran que en el antiguo Colesuyo, el estiércol era indispensable para lograr una mejor cosecha de los frutos. Un ejemplo de lo que decimos lo hallamos en un pasaje de la visita que en 1567 hizo Garcí Diez de San Miguel (1964 [1567]) a la provincia de Chucuito, donde el visitador señala, en su paso por el valle moqueguano, que los indios no podían...

[...] coger maíz sino es echando a la sementera que hacen cuando está algo crecido estiércol de pájaros de la costa de la mar que llaman guano porque sin ello no grana y esto lo traen veinte leguas de alli de unas islas que están dentro la mar [...] (p. 245).

Otra cuestión a considerar era el tipo y calidad del guano. En la mayoría de informes coloniales se hace mención del guano blanco, que es el más común en las islas. Sin embargo, también se debe considerar el guano rojo, que es una variedad que toma ese color por tener un mayor tiempo de exposición en la atmósfera (Sánchez García, 2016,p. 71).

Siguiendo una pesquisa de Edwin Adriazola (2015, p. 32), en el Archivo de Moquegua revisamos

[...] la extracción y saca de guanos de sementera y calidad rojo que llaman de Yquique, de un serro que yo el dicho don Alejo he descubierto en tierra firme inmediato al mar y punta de Cole, jurisdicción de la ciudad de Arica dibición del valle de Ylo [...] (ARM, escribano Joseph Fernández Dávila 1796, f. 49 r.).

\section{La posesión de las covaderas ${ }^{2}$}

Como lo advierte María Rostworowski, las islas guaneras de la costa peruana se caracterizaban por la falta de fuentes propias de agua potable, lo que las convertía en inhabitables para poblaciones permanentes (Rostworowski, 2005). Esto 1o comprobamos también en la costa moqueguana. Por citar un ejemplo, respecto a la parte de la Punta de Coles, se dirá que se...

[...] compone de peñas y riscos con una llanadita [que] consta inavitable desde el tiempo de la gentilidad [...] sin agua y las veses que se han ofrecido avitarlos pescadores para su mantenimiento han llevado el agua en vasijas desde el río de Hilo [...] (ART, Corregimiento, Causas Ordinarias, Leg. 1, Cua. 1, fol. 1r.).

Sobre la posesión de las covaderas Bente Bittmann (1986), citando a algunos cronistas, agrega que en la época incaica los depósitos de guano diseminados en las islas de la costa estaban sujetos a un "tipo de territorialidad", de manera que cada pueblo - y por lo tanto cada individuo - tenía derecho a una determinada parte de este recurso (p. 303). Así lo explica Garcilaso de la Vega (1609), que sobre el caso dice:

[...] cada isla estaba, por orden del Inca, señalada para tal o cual provincia, y si la isla era grande, la daban a dos o tres provincias $[\ldots]($ p. 240).

Sin embargo, con la imposición del sistema colonial y conforme los españoles empezaron a reconocer la importancia del guano para los cultivos, los indios empezaron a perder poco a poco la posesión de las islas guaneras. En un principio los colonos se hicieron con las islas temporalmente a través del arrendamiento de estas, luego, de manera arbitraria, prácticamente despojaron a los naturales de sus posesiones. El primer caso conocido se dio con los indios quinistacas que, debido a la erupción del volcán Huaynaputina, se habían asentado en el valle de Moquegua porque el de Quinistacas estaba "lleno de ceniza". Como consecuencia de su estancia, los indios habían contraído deudas debido a la tasa que tenían que cumplir con su encomendero, Andrés de Herrera y Castilla, vecino de Arequipa.

\footnotetext{
${ }^{2}$ Aunque el término covadera se usa en Bolivia y Chile: "Espacio de tierra de donde se extrae guano", lo utilizamos para este trabajo por motivos didácticos.
} 
Imposibilitados de poder pagar el tributo porque no recogían frutos, los indios no tuvieron más remedio que recurrir a su más preciado bien: las islas guaneras que tenían en la costa. Por esa razón, en 1615, los caciques quinistacas Cristóbal Segola y Pedro Acavana arrendaron a Bartolomé Martín de Quintanilla [...] las yslas del guano que tienen en la costa de la mar llamadas de Corocinto [...] (ARM, escribano Diego Dávila 1610-1615, f. 671r.).

Algunos años después ocurrió el segundo caso. Sucedió que el Protector de los Naturales de Arequipa, abogando por los indios de Puquina, Omate y Ubinas, se enfrentó a los hacendados del valle del Tambo, quienes habían privado a dichos indios de sus islas. En el juicio también intervino el vecino de Arequipa, Pedro Peralta de Rivera, quien asimismo - afirmaba tener posesión de las islas, argumentando que los indios sacaban el guano de las costas de Iquique y no de dichas islas. El proceso se volvió interminable con la presentación de probanzas y testigos (Rostworowski, 2005).

No obstante, en noticias posteriores tenemos constancia que algunas comunidades de indios continuaron posicionándose en las islas. Esto lo podemos observar durante la visita de Antonio Álvarez y Jiménez al pueblo de Puquina en 1792. Al respecto, el visitador reconoció que muchas islas eran poseídas por los pueblos indios, caciques y otros particulares, ya sea por costumbre antigua o por declaratorio de la Superioridad del Reino (Bitmman, 1986, p. 310). En tanto, Álvarez y Jiménez reconoce también que otras estaban en pleno litigio, como las islas...

[...] Perica y Blanca [que] quedaban a beneficio de la Comunidad de esta Doctrina [de Puquina], posteriormente y con razones fundadas he tenido por conveniente agregarle la Isleta nombrada la Blanca a Don Manuel Lajo Olim cacique de Urinsaya, mayormente cuando en este propio acto ha presentado un Escrito en que por sí mismo y a sus expensas ofrece continuar hasta su conclusión la Causa pendiente entre la Comunidad y el Coronel Don Domingo Bustamante sin más pensión que si se declara en favor de los indios se le ha de dar una de aquellas islas perdiendo sus costos si se declarase en contra [...] (Barriga, 1946, p. 277).
Tabla 2. Islas guaneras de las comunidades de Moquegua

\begin{tabular}{cc}
\hline \multicolumn{2}{c}{ Covaderas de las comunidades de Moquegua } \\
\hline Corocinto & Manza \\
\hline Los Frayles & Las Animas \\
\hline Empinadas & Pacay \\
\hline Perica & Hierba Buena \\
\hline Blanca & Caliyqui \\
\hline La Margarita & Chata \\
\hline Pocoguata (Pocoata) & Taranto \\
\hline Brava & Alfaro \\
\hline & De Jesús \\
\hline
\end{tabular}

Fuente: ARM, escribano Diego Dávila 1610-1615, f. 671r;

Barriga, 1946, p. 276-277; Rostworowski, 2005, p. 98.

Con la llegada de la República, la situación no cambió mucho, pero fue cuando se conoció las bondades del guano como fertilizante, que el Estado empezó a otorgarle interés a este recurso. Pese a que la comunidad puquina y otros pueblos seguían teniendo derechos sobre las islas guaneras a principios del siglo XIX (Cushman, 2018), tras tomar posesión de ellas, el Perú entró en 1840 en la era del guano, y las comunidades andinas perdieron poco a poco su influencia sobre este abono.

\section{Comentarios Finales}

Este estudio etnohistórico, que ha reconocido la importancia del guano en la historia del Perú, ha pretendido enfocarse en la pequeña región de Moquegua, un territorio que tiene la línea costera más corta del país y que ha demostrado - una vez más - el protagonismo de este recurso en las comunidades altoandinas. Si bien nosotros hemos dado a conocer lo indispensable de este fertilizante para la agricultura de los primeros pobladores de estas partes, queremos señalar también que la expropiación de las islas guaneras realizada por los hispanos no solo privó a los pobladores andinos de este preciado sustrato, sino también que sustituyó su valor casi sagrado por uno enteramente mercantil.

Hoy, queremos reconocer que si en efecto las covaderas de Moquegua ya no sufren la explotación de los mineros guaneros, no han dejado de perder importancia. Con el tiempo, se ha priorizado el valor turístico de lugares como Punta de Coles, que esconde una maravillosa belleza paisajística y uno de los puntos de encuentro más selectos de la fauna marina, sin dejar de lado su valor histórico, formado por varios siglos de actividad económica. 


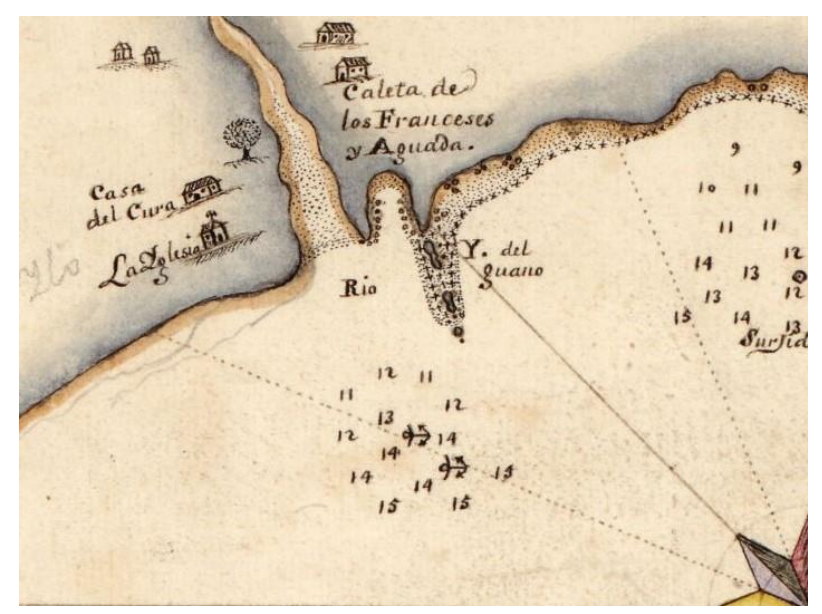

Figura 1. Mapa de Ilo (1933)

Fuente: LOC, Detalle de la Verdadera demostrasión del puerto de Ylo y de la caleta de Pacocha (1733).

\section{REFERENCIAS}

Adriazola Flores, E. (2015). Ilo: "Hitos de una economía colonial". Historia 2, 7-36. Arequipa: Ediciones Baluarte.

Asociación Contisuyo (1997). Contisuyo. Memoria de las Culturas del Sur. Moquegua: Asociación Contisuyo.

Barriga, V. (1946). Memorias para la Historia de Arequipa, Tomo II. Arequipa: La Colmena.

Bittman, B. (1986). Recursos naturales renovables de la costa del norte de Chile: Modos de obtención y uso. Etnografia e historia del mundo andino. Continuidad y cambio (Shozo Masuda Editor), 269-334. Tokio: Universidad de Tokio.

Cieza de León, P. (2005 [1553]). Crónica del Perú.
El señorio de los Incas. Caracas: Biblioteca Ayacucho.

Cushman, G. (2018). Los señores del guano. Lima: Instituto de Estudios Peruanos.

Diez de San Miguel, G. (1964 [1567]). Visita hecha a la provincia de Chucuito. Lima: Casa de la Cultura del Perú.

Garcilaso de la Vega, I. (2009 [1609]). Comentarios reales de los Incas. Arequipa: Biblioteca Juvenil Arequipa.

Pizarro, E. \& Soto, J. (2017). Explotación colonial de recursos naturales prehispánicos: el caso del huano de covaderas en el extremo norte de Chile (siglos XVI-XVII). Tránsitos historiográficos. Arica y su hinterland (siglos $X V I-X X)$ (Elías Pizarro Editor), 63-76. Arica: Universidad de Tarapacá.

Rostworowski, M. (2005). Recursos naturales renovables y pesca, siglos XVI-XVII/Curacas y sucesiones, costa norte. Lima: Instituto de Estudios Peruanos.

Sánchez García, Y. (2016). Caracterización química del guano de aves marinas de la Isla San Jerónimo, Baja California, México y su viabilidad como fertilizante agrícola. Ensenada: Tesis de Maestría del Centro de Investigación Científica y de Educación Superior de Ensenada.

Tantaleán Arbulú, J. (2011). La gobernabilidad y el leviatán guanero. Lima: Instituto de Estudios Peruanos.

\section{Fuentes de Archivo}

Archivo Regional de Moquegua (ARM)

Archivo Regional de Tacna (ART)

Fuentes Cartográficas

Biblioteca del Congreso de Estados Unidos/Library of Congress (LOC) 